\title{
Michael Stolleis Theodor Maunz - Ein Staatsrechtslehrerleben
}

Theodor Maunz (1901-1993) ist nun endgültig vom moralischen Eckstein zum Stein des Anstoßes der deutschen Staatsrechtslehre geworden. Was an undiskutierter und unverarbeiteter Vergangenheit teils verschüttet, teils abgearbeitet und vergeben schicn, tritt nun in abstoßender Farbc und Gestalt wieder hervor.

Dic Akten des "Falles Maunz"schienen geschlossen. Die Nachrufe auf den am ro. September 1993 verstorbenen, licbenswürdigen und zugleich verschlossenen Grcis waren geschrieben und enthielten nichts Überraschendes mehr. Im wescntlichen orienticrte man sich an der Laudatio, die Peter Lerche in der Festschrift des Vcrlags C. H. Beck "Juristen im Portrait " (1988) geschricben hatte. Dort hatte Lerche die seit dreißig Jahren einem breiteren Publikum bekannte NS-Vcrgangenheir von Maunz in einem einzigen, kunstvoll verhüllenden Satz an den Rand gedrängt. Schön war dies nicht, und es zeugte auch nicht von Freimut des Autors, aber immerhin lebte Maunz damals noch und es war kcine wissenschaftliche Untersuchung, sondern ein Beitrag zu einer "Festschrift 4 zu schreiben.

Soweit so gut. Professoren mit NS-Vergangenheit gab es dutzende, nienanden sonst freilich, der deswegen als Kultusminister spektakulär gestürzt war. Darüber hatte man um 1964 viel gesprochen, und später war es allmählich wieder vergessen worden. Für die jüngerc, damals studicrende Generation war der von Gerhard Hancy (Jena), Konrad Redeker und Hildegard Hamm-Brïcher ausgelöste "Fall Maunz « ein universitäres Enthüllungsstück, zusammen mit den Fällen Oberländer, Globke, Pölnitz, mit dem Auschwitz-Prozeß und dem Eichmann-Prozeß cin Stück Selbstfindung der Nachkriegsgeneration und somit auch "Beweisstück" im Generationenkonflikt. Das aus diesen Fällen und Prozessen genährte generellc Mißtrauen gegen Bekundungen und Vertuschungen der offiziellen Politik hat die Studentenbewegung von $1968 \mathrm{mit}$ in Gang gebracht.

Maunz war zum Exempel wie geschaffen: Sein solides, freilich nicht gerade inspirierendes Lchrbuch „Deutsches Staatsreche schien, wie der Volkswagen-Käfer, ein unverwüstliches Modell der Bundesrepublik - und wie der Volkswagen hatte es seine verborgenen Wurzcln im Dritten Reich. Der Grundgesetzkommentar »Maunz-Dürig« wurde sozusagen zum konstitutiven Orakel für den neuen Staat. „Papa Maunz» war cine Münchner Institution, vor allem beim Erwerb öffentlichrechtlicher Scheine. Er war sein unverglcichlicher Lehrer (und ein sehr milder Prüfer) «, wie Peter Lerche schricb und hinzufügte, Maunz habe »beinahe zuviel gütige Nachsicht... walten lassen $\aleph^{2}$. Güte, Höflichkeit, ungewöhnlichen Fleiß und Selbstdisziplin, Toleranz,

I K. Redeker, Bewalcigung der Vergangenheit als Aufgabe der Justrz, NJW 1964.

2 P. Lerche, Theodor Maun\%, in: Juristen im Portrait, Munchen 1988, 556. 
Praxisnähe, wissenschaftliche Vorurteilslosigkeit, alles dies besaß er. Niemand, der ihn gekannt hat, wird dies leugnen. "Kaum je gelang es jemandem bislang, den Hut zu einem früheren Zcitpunkt vor Maunz zu ziehen als dem, zu dem dieser seinen eigenen zog" (Lerche).

In größerem Abstand dürfte sich aber die Überzeugung durchsetzen, wissenschaftsgeschichtlich werde sich mit dem Namen Maunz trotz ciner viele Hunderte von Arbeiten umfassenden Bibliographie wohl kein wirklich eigenständiger Gedanke verbinden lassen. Man wird zugeben, daß scine das jeweils geltende Recht beglcitenden Beiträge kaum jemals origincll oder kühn waren, sondern geradezu als Inkarnation der mitrleren, konfliktvermeidenden herrschenden Meinung gelten konnten. "Sie zeichnen sich», wie die FAZ wieder einmal mit Feinsinn formulierte, »durch eine auf das Wesentliche konzentrierte, positivistisch getöntc Knappheit aus «3. Daß die "gütige Nachsicht« bei der Benotung studentischer Arbciten auch den angenchmen Effekt hattc, die Hörergelder in die Höhe zu treiben, könntc man wenigstens anmerken. Aber hiervon abgesehen war man sich bis vor kurzem einig: Maunz habe zwar eine NS-Vergangenheir gehabt, das lasse sich gar nicht leugnen, aber sie sei doch längst überwunden durch aktive Mitarbeit am demokratischen Gemeinwesen und durch redliche Ausarbeitung des der Bundesrepublik eigenen Staats- und Verwaltungsrechts. In pathctischer Stimmung konnte man vielleicht wirklich sagen: "Sein Name zählt zu den großen in der dcutschen Staatsrechtslehre «t und: "Die Rechtswissenschaft vom heutigen öffentlichen Recht kann man nicht feierlich loben, ohne Maunz zu loben...."

Einige charaktcristische Rätselfragen blieben freilich ungelöst. Schon 1934 ficl einem Rezensenten auf, Maunz habe seine »noch im vergangenen Jahr schroff gegensätzliche Einstellung "flink gewendet und sich mit einem "allzu zerknirschten pater peccavi« dem konkreten Ordnungsdenken Carl Schmitts in die Arme geworfen ${ }^{6}$. Der Privatdozent Maunz, der 1932 die Trennung von Recht und Politik vehement vertreten und gegen die "Untergrabung der Rechtsstellung des Individuums durch Zersetzung und Auflösung vorhandener Rechtssicherungen " gewettert hatte? ${ }^{7}$, wartete nun auf einen Lehrstuhl und betonte die politische Natur des Rechts und die Totalität des Führerwillens. Wie um absolute Linientreue zu bewveisen, trat Maunz ein Schüler jenes Hans Nawiasky, der seiner jüdischen Herkunft wegen schon vor 1933 in eine der größten antisemitischen Haßkampagnen geraten war ${ }^{8}-1936$ als Redner bei der Tagung "Das Judentum in der Rechtswissenschaft « auf und erläuterte dort »die verhängnisvolle Neigung jüdischer Verwaltungsrechtler zur liberalistischen Rechtsstaatsdoktrin «". 1937 kam dann endlich die Berufung auf den Freiburger Lehrstuhl. Der in Dachau geborene und mit Verwaltungserfahrung ausgestattete Maunz wußre, was es praktisch bedeutete, wenn er im Hörsaal "Gesetzmäßigkeit" durch "Rechtmäßigkeit" ersetzte, wenn er das "Ende des subjektiv-öffentlichen Rechrs" verkündete und die Verhaftungen durch die Gestapo zu justizfreien Hoheitsakten erklärte ${ }^{10}$. Daß er gleichzeitig seine Verbindungen zur Kirche nicht

3 F. K. Fromme, Theodor Maunz. gestorben, FAZ v. 11.9. 1993.

4 F. K. Fromme, a. a. O.

s Lerche, a. a. O., s60.

6 H. Rew $\beta$ zu Th. Mawnz, Neue Grundlagen des Verwaltungsrechts, Hamburg 1934, in: JW 1935, $496 \mathrm{f}$.

7 Th. Maunz, Hauptprobleme des öffentlichen Sachenrechts, $1933,316$.

$8 \mathrm{H}$. Zacher, Hans Nawiasky, in: Heinrichs u.a. ( $\mathrm{Hg}$.), Deutsche Juristen judischer Herkunft, 1993, $677-692$.

9 DJZ 1936, Sp. 1230 .

10 Th. Maunz, Gestalt und Reche der Polizei, 1943. Von den früheren Arbeiten siehe vor allem: Neue Grundlagen des Verwaltungsrechts, Hamburg 1934; Die Zukunft der Verwaltungsgerichrsbarkeit, DR 1935, 478; Die Enteignung in W/andel der Staatsauffassung, Freiburg 1936; Das Fnde des subjektivoffentlichen Rechts, ZgStW 96 (1936) 71 ff.; Verwaltung, Hamburg 1937. 
abreißen ließ und abseits der Freiburger Öffentlichkeit in einem Schwarzwalddorf die Sonntagsmesse besuchte, erwies sich als weitsichtige Sicherungsstrategie. Nachdem das NS-System Schiffbruch erlitten hatte, konnte Maunz auf die christlichsoziale Planke treten, als Gutachter in den "Kampf um den Südwest-Staat « eingreifen, bei der Beratung des Grundgesetzes mitwirken, das Lehrbuch "Deutsches Staatsrecht « verfassen, um endlich 1952 nach München berufen zu werden. Dort wurde Maunz zunächst »einfaches Mitglied « der CSU und bald darauf bayerischer Kultusminister. So war er dort wieder angekommen, wo er angefangen hatte, bei parlamentarischer Demokratic und Rechtsstaat.

II.

Was bislang als eine Karriere mit einem gehörigen Schuß Opportunismus, aber insgesamt doch dominanter fachlicher Solidität, enormem Fleiß, pädagogischer Begabung und Ncigung zu einer »konservativen Mittellage « bei jahrzehntelangem zweifelsfreiem Engagement für die parlamentarische Demokratie interpretiert werden mochte, sieht seit dem 24. September und dem 8. Oktober 1993 anders aus. An diesen Tagen enthüllte die "Deutsche National-Zeitung" (DNZ) des rechtsradikalen Führers der »Deutschen Volksunion «, Dr. Gerhard Frey" , daß Maunz seit 25 Jahren sein "wunderbarer Wegbegleiter " und "maßgeblicher Berater " gewesen war. Maunz habe, so die Deutsche National-Zeitung, hunderte von anonymen Artikeln für sie geschrieben, habe die Deutsche Volks-Union bei Programm- und Satzungsgestaltung beraten, habe kontinuierlich Rechtsgutachten zum Parteienrecht und Asylrecht, zum Vertrag von Maastricht, zum Postversand der Deutschen NationalZeitung und vielen anderen Themen erstellt. "Während der siebziger Jahre und lange darüber hinaus kam es allwöchentlich, zumeist am Montag, zu einer stundenlangen Besprechung aller zentralen politischen und juristischen Fragen zwischen Prof. Maunz und Dr. Frey " (DNZ v. 24.9.93). Die Sendung "Panorama (ARD) griff dic Sache auf und bat Rupert Scholz und Roman Herzog um Stellungnahmen. Die großen Zeitungen reagierten (ZEIT, Spiegel, Süddeutsche Zeitung, die Frankfurter Allgemeine freilich nur mit einer kleinen Agenturmeldung am Rande der Seite). Niemand zweifelt ernsthaft am sachlichen Kern der Mitteilung des Dr. Frey. Das Interesse Freys, die enge Beziehung zum bekanntesten Grundgesetz-Kommentator triumphierend auf den Tisch zu legen, ist klar. Deutsche National-Zeitung und Deutsche Volks-Union können sich sehen lassen, wenn der "größte Rechtsgelehrte Deutschlands" (DNZ v. 24.9. 1993) und »absolute Demokrat « (Scholz) ihnen viele Jahre lang beigestanden hat. Den Hinterbliebenen, unter ihnen sein Schwiegersohn, der Präsident des Bundesfinanzhofs Franz Klein ${ }^{12}$, sowie seinen Assistenten, Habi-

$11 \mathrm{Zu}$ dem gegen Frey angestrengten Verfahren gem. Art. 8 GG vgl. BVerfGE 38, 23-25.

12 Die Rolle des BFH-Prasidenten Prof. Dr. Franz Klean bei der Diskussion um das 75 -Jahres-Jubiläum des Reichsfinanzhofs/Bundesfinanzhofs in der Panorama-Sendung vom 9. September 1993 war wenig glucklich. Seine Äußerungen verdienen festgehalten zu werden: $\nsim$ ir sind diejenigen, die die Kontinuitait des Reiches, cla ging ja der Streit drum, ob die Bundesrepublik Kontinuitat des Reiches hatte, die führen wir beispielsweise durch "... "Da habe ich keine Bederıken, auch das zu begehen. Denn es ist eine große Zeit auch wahrend des Nationalsozialismus gewesen, wo sachlich Kecht gesprochen wurde. Und es sind dann Urteile da, die unheimlich sind und dié auch als unheimlich bezeichnet werden, auch von mir als unheimlich bezeichnet worden sind .... . I Ich freue mich auf meinc Feier, und ich lasse mir die Feier auch niche von denjenigen, die meinen, weil sie nicht eingeladen sind, vermiesen. Auch diejenigen, die heute Vorwurfe machen, haben wahrscheinlich die HJ-Uniform getragen $\alpha$. Vgl. dazu G. Felix, Der Reichsfinanzhof im "Drıtcen Reıcha, die jüdischen Deutschen und die unbegrenzte Auslegung, in: Betriebsberater 1993, 1297-1303; K. Tipke, Über die Juden-Rechtsprechung des RFH, in: Betriebsberater $1993,1813 \mathrm{ff}$. 
litanden und Doktoranden mag die Enthüllung peinlich sein. Das ficht Herrn Dr. Frey nicht an. Er ist nicht der Mann, der auf solche Empindlichkeiten und Berührungsängste Rücksicht nimmt. Sein Kalkül zielt darauf, durch systematische Grenzverwischung vom rechten Rand in die konservativc Mitte zu rücken. Hierfür war das Engagement von Maunz cin willkommenes Geschenk. Es macht dic Neonazis rcputierlicher, wenn sie zeigen können, daß es ihnen gelungen ist, sozusagen schon in den Vorhof der Vereinigung der deutschen Staatsrechtslehrer eingedrungen zu sein.

Die Deutungen jener Symbiose zwischen Maunz und Frey oszillieren noch zwischen positiv und negativ besetzten Mustern: Maunz habe "nicht Nein sagen können «, der alte Herr sei viellcicht "auch etwas mißbraucht « worden (Scholz). Der durch seincn Vcrstand rechtsradikaler Bücher, Platten und Videos wohlhabende Frey habe vermutlich auch für alle Bcratungs- und Schriftstellerdienste gut gezahlt. Zudem habe Frey wohl dem gcstürzten Kultusminister Maunz Seelenbalsam verabreicht, indem er jene Affaire von 1964 als Werk einer linken Pressekampagne dargestellt habe. Diese Deutungen mögen alle einzeln oder kumulativ zutreffen. Interessanterweisc hat abcr bisher nicmand behauptet, es handle sich um einen Fall ungebrochener Kontinuität rechtsradikalen Denkens seit r933. So cinfach liegen die Dinge in der Tat nicht. Man kommt dem Typus des Juristen Maunz wohl näher, wenn man seine Aktivitäten - quer durch wechselnde Regime - unter dem Aspekt der Optimierung der Lebens- und Wirkensbedingungen betrachtct. Maunz war Vernunftrcpublikaner und Rechtsstaatler, als dies am Ende der Weimarer Republik noch zum traditionellen Verhaltenskodex gchörtc, cr war Nationalsozialist, solangc cs dic anderen waren und noch ein bißchen mehr und länger, dann wieder naturrechtlich garnierter Rechtspositivist, Demokrat und Föderalist, je nach Zeit und Umgcbung, so wie das Chamäleon oder die Wechselblütler die Gabe besitzen, Farbe und Temperatur der Umgebung anzupassen. Letzteres natürlich nur inncrhalb ciner gewissen Bandbreite. Man darf annchmen, daß dic von Maunz in der National-Zeitung anonym gcschriebenen Artikel seine wirkliche, tiefinnerste Meinung wiedergcben. Er schricb anonym und er war als Emeritus den Zwängen cnthoben, irgend jemandem nach dem Munde reden zu müssen. Daß er diese tiefinnerste Meinung unter striktcr Gcheimhaltung publizierte, spricht weniger für ein rudimentäres Unrechtsbewußtsein als für ein fein entwickeltes Sensorium dafür, wicviel man der Öffentlichkeit und der Kollegenschaft zumuten konnte. Seine Witterung für Tabus ließ ihn auch hicr den zu erwartenden Konflikt scheuen. Maunz blieb sich treu: In der NS-Zeit an den Sonntagen "heimlich « in die Messe, in der Bundesrepublik an den Montagen "heimlich " zu den Rechtsradikalen. Doppelleben als Optimierungsstrategie.

Wo aber liegt, jenscits der Unappetitlichkeit des ganzen Vorgangs, das moralische Problem für die deutsche Staatsrcchtslehrc? Es licgt, so schcint mir, nicht bci Maunz und seincm Bild in der Wissenschaftsgeschichtc, sondern in der Staatsrechtslehrerschaft als Korporation. Vorgänge wie diese werden in der Vereinigung Deutscher Staatsrechtslehrer nicht diskutiert, ja wohl nicht einmal als diskussionwürdig empfunden. Die Verantwortung des Öffentlichrechtlers als Rechtslehrer, Forscher, Gutachter und Prozeßvertreter vor dem Hintergrund solcher Erfahrungen - das wäre ein Thema. Aber diese Büchse der Pandora zu öffnen, wagt kcin Vorstand jener Vereinigung. So wird dcm aufmerksamen Ohr noch eine Wcile verlegenes Gctuschel vernehmbar sein. Danach dürfe sich wieder brütendes Schweigen über die Zunft legen. 